\title{
Atypical diffuse bilateral cystic lung changes secondary to erlotinib treatment in a patient with metastatic non-small cell lung carcinoma: A case report and literature review
}

\author{
JAWAHER ANSARI ${ }^{1}$, ENAS BATUBARA ${ }^{2}$, MUHAMMAD ALI $^{1}$, ASHRAF FARRAG $^{1,4}$, \\ FARHAT BASHIR $^{1}$, HUSSEIN R. FARGHALY ${ }^{3}$, ARWA M. ALI ${ }^{1,5}$ And ARIF SHAUKAT ${ }^{1}$ \\ Departments of ${ }^{1}$ Oncology and ${ }^{2}$ Pulmonology, ${ }^{3}$ Nuclear Medicine Division, Prince Sultan Military Medical City, \\ Riyadh 11159, Saudi Arabia; ${ }^{4}$ Clinical Oncology Department, Assiut University Hospital, Assiut University; \\ ${ }^{5}$ Medical Oncology Department, South Egypt Cancer Institute, Assiut University, Asyut 71515, Egypt
}

Received November 15, 2017; Accepted May 2, 2018

DOI: $10.3892 / \mathrm{mco} .2018 .1620$

\begin{abstract}
Erlotinib is a first-generation epidermal growth factor receptor (EGFR) tyrosine kinase inhibitor (TKI) approved in the first-line treatment of advanced non-small-cell lung cancer (NSCLC) patients with sensitizing epidermal growth factor receptor (EGFR) mutations. The response rate to erlotinib is $\sim 60 \%$ and the incidence of erlotinib-induced interstitial lung disease (ILD) is $\sim 1-4 \%$. The Response Evaluation Criteria in Solid Tumours (RECIST) tool is commonly used to assess response to erlotinib; however, evaluation of response and subsequent progression in the presence of atypical cystic lung changes may be challenging. We herein present a rare case of diffuse cystic lung changes secondary to erlotinib treatment in a patient with EGFR mutation-positive metastatic NSCLC. Challenges in assessing atypical tumour response to erlotinib, pitfalls in using RECIST and differential diagnosis of TKI-related ILD are discussed in detail.
\end{abstract}

\section{Introduction}

Lung cancer is the most common cause of cancer-related mortality worldwide, responsible for nearly 1.59 million deaths in 2012 (1). Over the last decade, molecular translational research advances have led to the identification of genetic alterations, such as epidermal growth factor receptor (EGFR) mutations, leading to pathway-directed systemic therapy (2). Erlotinib is an EGFR tyrosine kinase inhibitor (TKI) that potently inhibits the intracellular phosphorylation of EGFR, resulting in cell stasis and apoptosis. For NSCLC patients

Correspondence to: Dr Jawaher Ansari, Department of Oncology, Prince Sultan Military Medical City, P.O. Box 7897, Riyadh 11159, Saudi Arabia

E-mail: jak.ansari@gmail.com

Key words: non-small-cell lung cancer, erlotinib, Tarceva, cystic lung, interstitial lung disease, epidermal growth factor receptor with sensitizing EGFR mutations, such as exon 19 deletion or exon 21 point mutation, treatment with erlotinib has achieved improvements in response rate and progression-free survival when compared to chemotherapy in several randomized controlled trials, and is currently a recommended treatment option (3).

The Response Evaluation Criteria in Solid Tumours (RECIST) tool is typically used for assessment of treatment response to TKIs. The aim of the present study was to report an interesting case of a patient with metastatic NSCLC who developed extensive cystic lung changes following treatment with erlotinib, and discuss the dilemma in assessing atypical tumour response to TKI against the possibility of TKI-related interstitial lung disease (ILD).

\section{Case report}

A 57-year-old female patient presented with a 6-month history of productive cough and breathlessness on moderate exertion. The patient's past medical history included hypertension, irritable bowel syndrome and treated brucellosis 18 months prior to presentation. She was a lifelong non-smoker and the family history was unremarkable. The patient's World Health Organisation performance status score was 1. Clinical examination revealed bronchial breathing over the right lung. Sputum culture and polymerase chain reaction assay ruled out the possibility of tuberculosis or other infections. Serology for brucella antibodies was negative. A computed tomography (CT) scan revealed diffuse right lung consolidation, mediastinal lymphadenopathy, bilateral lung nodules and multiple sclerotic bone metastases. Bronchoscopy-guided biopsy from the right lung revealed invasive adenocarcinoma with positive staining for thyroid transcription factor-1, cytokeratin 7 and napsin $\mathrm{A}$, consistent with primary lung adenocarcinoma.

The patient received palliative chemotherapy with carboplatin area under the curve 5 and pemetrexed $500 \mathrm{mg} / \mathrm{m}^{2}$ intravenously every 21 days after receiving vitamin B12 and folic acid pre-medication. A follow-up CT scan after 3 cycles of chemotherapy revealed disease progression, 
with an increase in the size and number of bilateral lung metastases (Fig. 1). Molecular testing results subsequently revealed the presence of EGFR exon 19 deletion and the treatment was switched to erlotinib $150 \mathrm{mg}$ once daily. A CT scan after 3 months of erlotinib treatment revealed extensive cystic changes in both lungs, replacing the previously seen widespread metastatic lung nodules (Fig. 2A). Clinically, the patient did not complain of either cough or breathlessness, and on examination there was evidence of diffuse reduction in bilateral lung air entry. Differential diagnosis of the CT changes included either erlotinib-associated ILD or atypical treatment response to erlotinib. Initial investigations were aimed at ruling out other pulmonary diseases that could mimic the clinical presentation. Pulmonary function tests (PFTs) revealed moderate obstruction with no bronchodilator effect. The predicted forced expiratory volume in $1 \mathrm{sec}$ was $69.4 \%$ and the predicted forced vital capacity was $80.2 \%$. Taking into consideration the diffuse cystic lung changes, transbronchial biopsy was not attempted due to the risk of pneumothorax. Pleural tap was performed, which ruled out the possibility of chylothorax. The pleural fluid lactate dehydrogenase (LDH) (147 U/1)/serum LDH (202 U/l) ratio was 0.72 , and the pleural fluid protein $(44 \mathrm{~g} / 1) /$ serum protein $(63 \mathrm{~g} / \mathrm{l})$ ratio was 0.69 , suggesting the fluid to be an exudate. Culture of the pleural fluid failed to grow any organisms. Follow-up CT scans after 10 months (Fig. 2B) and 20 months (Fig. 2C) of erlotinib treatment revealed progressive worsening of the cystic lung changes. Fluorine-18 fluorodeoxyglucose positron emission tomography (PET)-CT performed 12 months after commencement of erlotinib treatment revealed no metabolic activity in the diffuse bilateral cystic lung changes, making ILD or disease progression less likely (Fig. 3). Four months later, the patient was commenced on monthly zoledronic acid infusions in addition to erlotinib. Follow-up serial PFTs revealed no deterioration 12 months after commencement of erlotinib.

Despite progressively worsening extensive bilateral cystic lung changes on erlotinib, response to treatment is considered to be the most likely explanation, given the symptomatic improvement, stable PFTs and lack of PET avidity in the cystic changes. The patient also developed erlotinib-related grade 2 acneiform skin rash, for which she received a 3-month course of doxycycline with good symptom relief. The patient has been on erlotinib treatment for 22 months and it was decided to continue with this treatment until the development of radiological disease progression or worsening of the respiratory symptoms. The last follow-up appointment with the patient was conducted on 21st March 2018.

\section{Discussion}

Cystic lung changes secondary to erlotinib treatment have only been previously described in a single case report. Sakhri et al (4) reported the case of a 35-year-old woman with metastatic lung adenocarcinoma who developed acute respiratory distress syndrome secondary to bilateral spontaneous pneumothorax and pulmonary cystic changes after 2 months of erlotinib treatment. In comparison, our patient has a prolonged duration of response to erlotinib, despite exhibiting significantly more extensive bilateral cystic lung

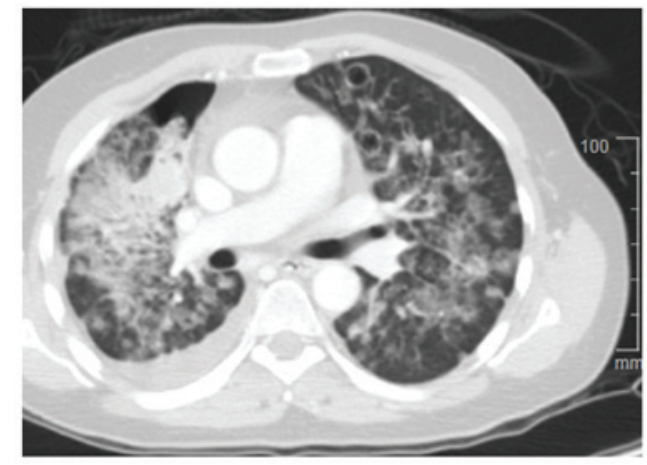

Figure 1. Axial contrast enhanced computed tomography scan of chest prior to erlotinib treatment showing bilateral multiple lung metastases.
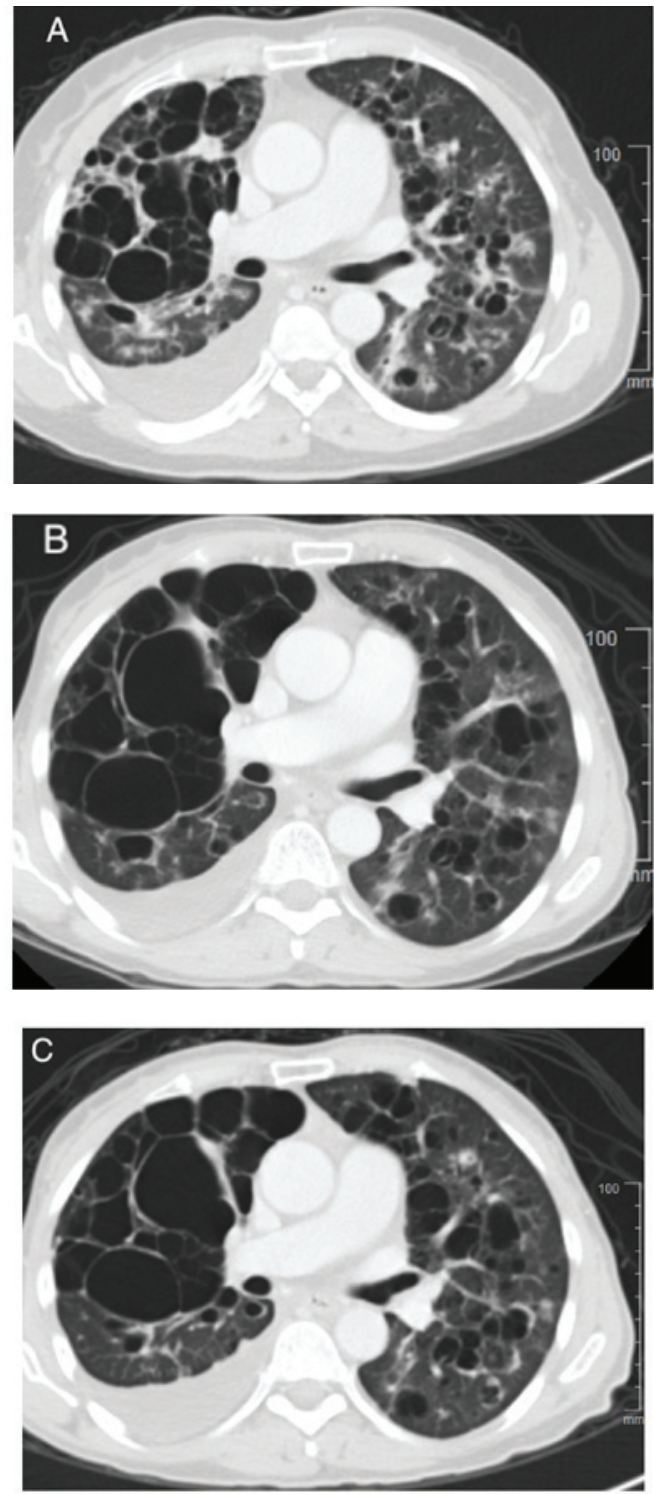

Figure 2. Axial contrast enhanced computed tomography scan of chest after (A) 3 months, (B) 10 months and (C) 20 months of erlotinib treatment showing cystic transformation of bilateral lung metastases with progressive worsening.

changes. Gefitinib, another first-generation EGFR-TKI, has also been reported to cause pulmonary cystic changes (5). 


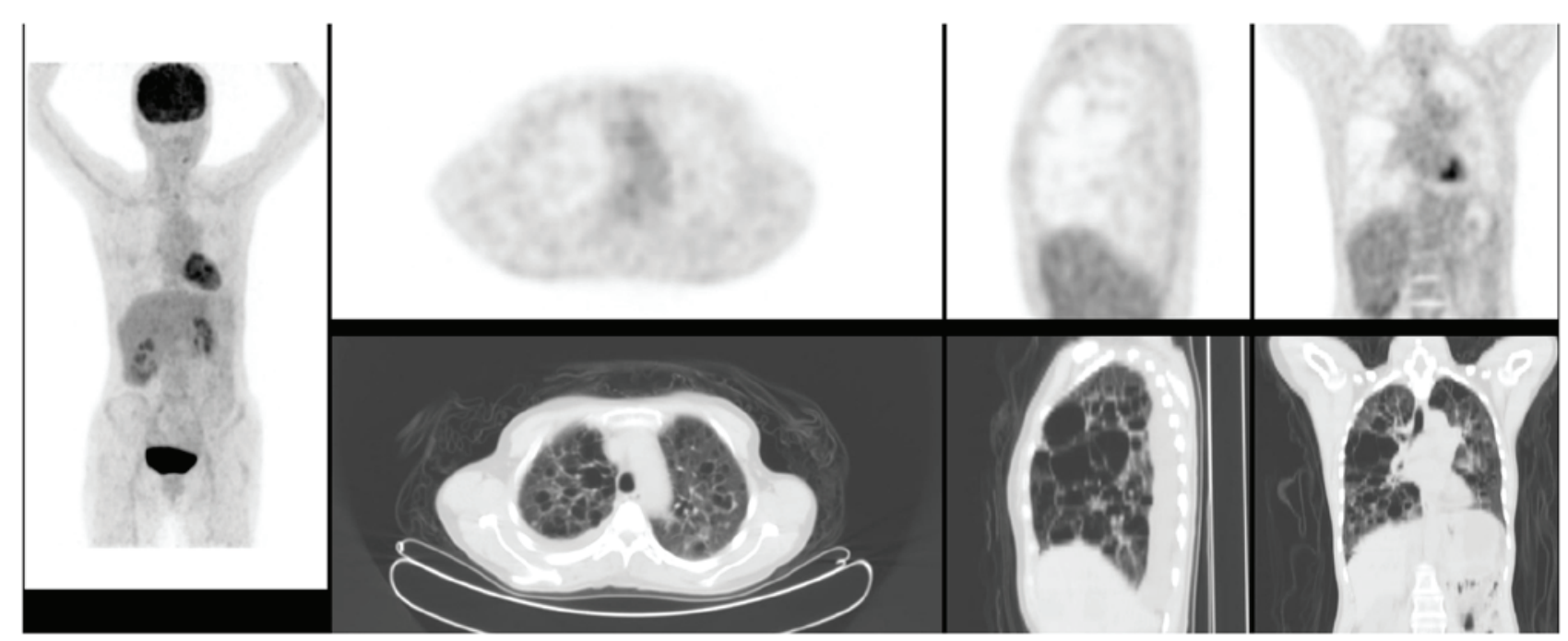

Figure 3. ${ }^{18} \mathrm{~F}$-Fluorodeoxyglucose positron emission tomography-computed tomography performed 12 months after commencement of erlotinib treatment, showing absence of metabolic activity in the diffuse bilateral cystic lung changes.

Therefore, it is plausible that cystic transformation of NSCLC metastases may be a class effect of EGFR-TKIs. It has been hypothesized that the anti-angiogenic effects of TKIs, or possibly tumour lysis of metastatic deposits, may lead to cavitation or cystic transformation of metastatic deposits. The effect of EGFR inhibition on type 2 pneumocytes has also been proposed as a cause for the cystic lung changes. EGFR is highly expressed on human alveolar epithelial type II pneumocytes, participating in the repair of alveolar walls (6). EGFR-TKIs may hinder the repair of alveolar damage, leading to lack of regeneration of lung parenchyma and, consequently, cystic lung changes (7).

Intriguingly, pulmonary adverse events, including ILD, have been described in erlotinib-treated NSCLC patients. In the pivotal BR 21 study in patients with NSCLC, the incidence of ILD was $0.8 \%$ in both the placebo and erlotinib groups (8). However, in the phase 4 POLARSTAR study evaluating the safety and efficacy of erlotinib in 9,909 Japanese patients with advanced NSCLC, the incidence of ILD was $4.3 \%$ (9). A multivariate analysis identified the following baseline risk factors associated with the development of ILD in erlotinib-treated patients: Pre-existing/concurrent ILD, chronic obstructive pulmonary disease or emphysema, lung infection, pre-existing pulmonary fibrosis, limited residual normal lung, smoking history, poor performance status, and $<360$ days from cancer diagnosis to the initiation of erlotinib. The typical $\mathrm{CT}$ findings in erlotinib-induced ILD include extensive bilateral ground glass attenuation and alveolar air space densities $(10,11)$. Distinguishing between erlotinib-related cystic lung changes and erlotinib-associated lung toxicity may be challenging, particularly if the patient develops respiratory symptoms.

RECIST entails unidimensional anatomical assessment of tumour burden and is a widely-used tool for assessment of tumour response in clinical trials (12). However, as it was originally designed to assess cytotoxic chemotherapy response, RECIST may not capture all the patterns of response and progression observed with TKIs. It is well recognised that, for gastrointestinal stromal tumours treated with the TKI imatinib, decrease in tumour attenuation on CT is more likely to represent a response to treatment rather than reduction in tumour dimensions (13). Several new response criteria have been proposed to capture the diverse nature of responses observed with TKIs, but these are yet to be prospectively validated (14).

In our patient, a time of $<360$ days from diagnosis to erlotinib treatment appears to be the only risk factor for erlotinib-induced ILD. The clinical and radiological findings are more in keeping with treatment response rather than erlotinib-induced ILD. Continuation of erlotinib in this patient could risk progressive worsening of the cystic lung changes with the consequent risk of respiratory failure. Cessation of erlotinib treatment may halt the worsening of the cystic lung changes, but is unlikely to lead to a reversal of these changes, whereas it would be associated with a significant risk of disease progression. As the patient had prior disease progression on chemotherapy and is currently clinically stable on erlotinib, the risk-benefit ratio would be in favour of continued treatment with erlotinib. If the patient develops any deterioration of her respiratory symptoms, switching to a second-generation TKI, such as afatinib, will be considered.

Cystic transformation of lung metastases may represent a class effect of EGFR-TKIs in a selected subset of patients. Evaluation of response and subsequent progression in the presence of cystic lung changes may be challenging. There is a clear need for the development of more standardized and reproducible methods of tumour response assessment for EGFR-TKIs, potentially accounting for the functional changes and cystic transformation of metastases. Erlotinib-induced ILD is a well-recognised adverse event and must be considered in the differential diagnosis of atypical lung imaging after drug initiation.

\section{Acknowledgements}

Not applicable. 


\section{Funding}

No funding was received.

\section{Availability of data and materials}

Not applicable.

\section{Authors' contributions}

JA and EB conceived and designed the study. JA, AF, MA, AMA and EB drafted the manuscript. MA, AF, FB, HRF, AMA and AS revised the manuscript for important intellectual content and provided final approval of the version of the manuscript to be published. JA and EB supervised the study.

\section{Ethics approval and consent to participate}

Not applicable.

\section{Consent for publication}

Written informed consent for publication has been obtained from the patient.

\section{Competing interests}

The authors declare that they have no competing interests.

\section{References}

1. Ferlay J, Soerjomataram I, Ervik M, Dikshit R, Eser S, Mathers C, Rebelo M, Parkin DM, Forman D and Bray F: GLOBOCAN 2012 v1.0, cancer incidence and mortality Worldwide: IARC CancerBase No. 11 [Internet]. International agency for research on cancer. Lyon, 2013. http://globocan.iarc.fr. Accessed March 21, 2018
2. Yarden Y and Sliwkowski MX: Untangling the ErbB signalling network. Nat Rev Mol Cell Biol 2: 127-137, 2001.

3. Novello S, Barlesi F, Califano R, Cufer T, Ekman S, Levra MG, Kerr K, Popat S, Reck M, Senan S, et al: Metastatic non-small-cell lung cancer: ESMO clinical practice guidelines for diagnosis, treatment and follow-up. Ann Oncol 27 (Suppl 5): v1-v27, 2016.

4. Sakhri L, Meynet E, Ferrer L, Pirvu A, Ferretti G and Moro-Sibilot D: Atypical response to erlotinib in a patient with metastatic lung adenocarcinoma: A case report. J Med Case Rep 8: 335, 2014.

5. Ryu YJ, Chun EM, Lee SN and Shim SS: Progressive multiple cystic changes in both lungs in a patient treated with gefitinib for lung adenocarcinoma with multiple lung metastases. Korean J Radiol 15: 300-304, 2014

6. Baybutt RC, Smith BW, Donskaya EV, Hu L, Li T and Wang W: The proliferative effects of retinoic acid on primary cultures of adult rat type II pneumocytes depend upon cell density. In Vitro Cell Dev Biol Anim 46: 20-27, 2010.

7. Higenbottam T, Kuwano K, Nemery B and Fujita Y: Understanding the mechanisms of drug-associated interstitial lung disease. Br J Cancer 91 (Suppl 2): S31-S37, 2004.

8. Shepherd FA, Rodrigues Pereira J, Ciuleanu T, Tan EH, Hirsh V, Thongprasert S, Campos D, Maoleekoonpiroj S, Smylie M, Martins R, et al: Erlotinib in previously treated non-small-cell lung cancer. N Engl J Med 353: 123-132, 2005.

9. Lind JS, Smit EF, Grünberg K, Senan S and Lagerwaard FJ: Fatal Interstitial lung disease after erlotinib for non-small cell lung cancer. J Thorac Oncol 3: 1050-1053, 2008

10. Vahid B and Esmaili A: Erlotinib-associated acute pneumonitis: Report of two cases. Can Respir J 14: 167-170, 2007.

11. Chou CL, Ko HW, Wang CW, Yu CT, Kuo HP and Huang CD: Erlotinib-associated near-fatal interstitial pneumonitis in a patient with relapsed lung adenocarcinoma. Chang Gung Med J 33: 100-105, 2010.

12. Eisenhauer EA, Therasse P, Bogaerts J, Schwartz LH, Sargent D, Ford R, Dancey J, Arbuck S, Gwyther S, Mooney M, et al: New response evaluation criteria in solid tumours: Revised RECIST guideline (version 1.1). Eur J Cancer 45: 228-247, 2009.

13. Choi H, Charnsangavej C, Faria SC, Macapinlac HA, Burgess MA, Patel SR, Chen LL, Podoloff DA and Benjamin RS: Correlation of computed tomography and positron emission tomography in patients with metastatic gastrointestinal stromal tumor treated at a single institution with imatinib mesylate: Proposal of new computed tomography response criteria. J Clin Oncol 25: 1753-1759, 2007.

14. Lee HY, Lee KS, Ahn MJ, Hwang HS, Lee JW, Park K, Ahn JS, Kim TS, Yi CA and Chung MJ: New CT response criteria in non-small cell lung cancer: Proposal and application in EGFR tyrosine kinase inhibitor therapy. Lung Cancer 73: 63-69, 2011. 